\title{
Chromatic discrimination in the presence of incremental and decremental rod pedestals
}

\author{
DINGCAI CAO, ${ }^{1}$ ANDREW J. ZELE, ${ }^{2}$ AND JOEL POKORNY ${ }^{1}$ \\ ${ }^{1}$ Visual Science Laboratories, Department of Ophthalmology and Visual Science, The University of Chicago, Chicago, Illinois \\ ${ }^{2}$ School of Optometry and the Institute of Health and Biomedical Innovation, Queensland University of Technology, Brisbane, \\ Australia
}

(Received October 1, 2007; ACCEPTEd February 18, 2008)

\begin{abstract}
Signals from rods can alter chromatic discrimination. Here, chromatic discrimination ellipses were determined in the presence of rod incremental and decremental pedestals at mesopic light levels. The data were represented in a relative cone Troland space, normalized by discrimination thresholds measured along the cardinal axes without a rod pedestal. In the quadrant of cone space where L-cone relative to M-cone excitation increased, and S-cone excitation decreased, rod incremental pedestals degraded chromatic discrimination, and rod decremental pedestals improved chromatic discrimination. Discrimination in the other three quadrants of cone space was unaffected by the incremental or decremental rod pedestals. A second experiment measured chromatic discrimination under conditions where cone pedestals were matched to the appearances of the incremental and decremental rod pedestals. Based on the matching pedestal data, discrimination then could be measured independently along the cardinal axes using either chromatic $[\mathrm{L} /(\mathrm{L}+\mathrm{M}) ; \mathrm{S} /(\mathrm{L}+\mathrm{M})]$ or luminance $(\mathrm{L}+\mathrm{M})$ pedestal components. The discrimination data altered by the rod pedestals were similar to chromatic cone pedestals for $\mathrm{L} / \mathrm{M}$ increment discrimination, but similar to luminance cone pedestals for $\mathrm{S}$ decrement discrimination. The results indicated that the rod and cone signals combined differently in determining chromatic discrimination for different post-receptoral pathways.
\end{abstract}

Keywords: Chromatic discrimination, Rod pedestal, Mesopic, Rod-cone interaction, Post-receptoral pathways

\section{Introduction}

At mesopic light levels, where both rods and cones are operating, rod activity is reported to degrade chromatic discrimination in trichromatic observers (for a review see Buck, 2004). This has been attributed to a desaturation effect from rods that leads to a weakened chromatic signal (Lythgoe, 1931; Gilbert, 1950). This interpretation however, raises several issues. First, rod excitation does not always lead to a desaturated appearance of a test field, as the chromaticity shift caused by increased rod activity can be in directions other than toward white (Cao et al., 2005). Second, rod excitation can improve wavelength discrimination at longer wavelengths (Stabell \& Stabell, 1977b).

The purpose of this study is to characterize the effect of rod activity on chromatic discrimination at mesopic light levels. The experiments were conducted using a four-primary photostimulator to provide independent control of the stimulation of rods and three cone photoreceptor types (Pokorny et al., 2004) at a fixed retinal locus, and under the same adaptation conditions for both the rod and cone measurements. There were two experiments. In the first,

Address correspondence and reprint requests to: Joel Pokorny, Visual Science Laboratories, The University of Chicago, 940 East 57th Street, Chicago, IL 60637. E-mail: j-pokorny@uchicago.edu chromatic discrimination ellipses were measured in the presence of a $20 \%$ rod incremental or a $20 \%$ rod decremental pedestal contrast and compared to the discrimination ellipses measured without a rod pedestal. Because a change in rod signals in the test area are known to alter the chromaticity and brightness of the test (Cao et al., 2005), the second experiment was designed to determine if the rod pedestals altered chromatic discrimination by rod contributions to the chromatic and/or luminance pathway.

\section{Material and methods}

\section{Apparatus}

A two-channel four-primary photostimulator, which allows independent control of excitation of the rods and three cone types independently (Shapiro et al., 1996), generated a center-surround stimulus configuration. A complete description of the design of the photostimulator is given by Pokorny et al. (2004) and an example of its implementation is detailed in Cao et al. (2005, 2006, 2007). The radiances of the primaries (dominant wavelengths: 459, 561, 516 , and $658 \mathrm{~nm}$ ) were controlled by amplitude modulation of a 20 $\mathrm{kHz}$ carrier fed by an eight-channel analog output Dolby soundcard (M-Audio-Revolution 7.1 PCI) with a 24 bits digital-to-analog converter (DAC) operating at a sampling rate of $192 \mathrm{kHz}$. The output 
of the DAC was demodulated (Puts et al., 2005) and sent to voltageto-frequency converters that provided $1 \mu$ s pulses at frequencies up to $250 \mathrm{kHz}$ to control the LEDs (Swanson et al., 1987). The soundcard with demodulator has a precision of greater than 16 bits (Puts et al., 2005). All stimuli were generated using custom developed software running on a Macintosh G5 PowerPC computer.

\section{Calibration procedures}

The photostimulator was calibrated in two steps. The first pertained to the measurement of the spectral distribution and the linearization of physical light for each LED. The second involved observer calibrations to compensate for individual differences in pre-receptoral filtering and receptoral spectral sensitivities. Details of the calibration procedures have been described (Sun et al., 2001; Pokorny et al., 2004; Cao et al., 2005).

\section{Observers}

Two experienced psychophysical observers, the authors AJZ and DC, participated in the study. Both observers have normal color vision (assessed by the Neitz OT anomaloscope) and hue discrimination (assessed by the Farnsworth-Munsell 100-hue test). A refractive correction lens was placed on the instrument side of the $2 \mathrm{~mm}$ artificial pupil for DC. Data were collected at Queensland University of Technology, Australia, for AJZ, and at the University of Chicago, USA, for DC. The instrument setup and calibration procedures were the same in both laboratories. The Institutional Review Boards at The University of Chicago and Queensland University of Technology approved all experimental procedures.

\section{Stimuli}

The stimulus was a $2^{\circ}$ circular field set within a $13^{\circ}$ surround. A white fixation light placed in the center of the stimulus at $7.5^{\circ}$ temporal eccentricity. The center and surround fields had a chromaticity metameric to the equal-energy-spectrum with a retinal illuminance of two photopic Td. The chromaticity was defined in a relative cone Troland space (Smith \& Pokorny, 1996), which is the MacLeod \& Boynton (1979) physiological cone excitation space with the $\mathrm{S} /(\mathrm{L}+\mathrm{M})$ value for an equal-energy-spectrum light (EES) normalized to 1.0. The cardinal axes in the cone space reflect post-receptoral spectral processing in the Parvocellular (PC-) and Koniocellular (KC-) pathways. Chromatic discrimination was measured in the presence of rod pedestals (Experiment 1), or cone pedestals that perceptually matched the rod pedestals (Experiment 2 ) in the $2^{\circ}$ center with a steady surround, and compared to the data measured without a pedestal. During the stimulus presentation, an equiluminant change in cone stimulation along one of eight angles in the cone space was pulsed simultaneously with the rod or cone pedestal in one of the three temporal intervals (Fig. 1). Each $400 \mathrm{~ms}$ test stimulus interval was separated by a $500 \mathrm{~ms}$ inter stimulus intervals during which time the center and surround were isomeric. A pilot study measuring chromatic discrimination as a function of stimulus duration without the pedestal indicated that a $400 \mathrm{~ms}$ duration was sufficient to return a minimum discrimination threshold along the $\mathrm{L} /(\mathrm{L}+\mathrm{M})$ and $\mathrm{S} /(\mathrm{L}+\mathrm{M})$ axes.

\section{Procedure}

Prior to the start of each session, the observer dark-adapted for $30 \mathrm{~min}$ to ensure maximal rod sensitivity. Chromatic discrimi- nation was determined using a three-alternative forced choice double staircase procedure, with one staircase for incremental discrimination and the other for decremental discrimination. In each trial of a session, the cone stimulation change along one axis in the cone space was presented in one of the three intervals. A beep was sounded in the beginning of each interval. The observer identified the interval and direction of the cone stimulation changes, by pressing buttons on a gamepad sensed by the computer. The computer provided a feedback signal by generating two sounds, one for correct response and one for a wrong response. The staircase procedure continued until 10 reversals occurred. The values of the last six reversals were averaged as the threshold of that session. Each angle for each condition was repeated four to six times on different days with the mean threshold and standard error reported.

\section{Experiment 1: Chromatic discrimination in the presence of rod pedestals}

Chromatic discrimination was measured for three conditions: (1) no pedestal: center and surround had identical rod stimulation, (2) rod incremental pedestal: rod stimulation in the center was pulsed as a $20 \%$ Weber contrast increment, and (3) rod decremental pedestal: the rod stimulation in the center was pulsed as a $20 \%$ Weber contrast decrement. Initially, chromatic discrimination thresholds along the cardinal axes $\left(0^{\circ}, 90^{\circ}, 180^{\circ}, 270^{\circ}\right)$ in the cone space for the no pedestal condition were determined. A normalized cone space was defined with the measured thresholds for the no pedestal condition along the cardinal axes representing unit distances from the origin (Nagy et al., 1987). In the normalized cone space, the first or third quadrants show co-varying $\mathrm{L} /(\mathrm{L}+\mathrm{M})$ and $\mathrm{S} /(\mathrm{L}+$ $\mathrm{M}$ ) in the same polarity, leading to more purplish (the first quadrant) or yellow-greenish (the third quadrant) percepts. The second or fourth quadrants show co-varying $\mathrm{L} /(\mathrm{L}+\mathrm{M})$ and $\mathrm{S} /(\mathrm{L}+\mathrm{M})$ in an opposite polarity, leading to more blue-greenish (the second quadrant) or orangish (the fourth quadrant) percepts. In the normalized cone space, discrimination was measured along the diagonal axes $\left(45^{\circ}, 135^{\circ}, 225^{\circ}, 315^{\circ}\right)$ for the no pedestal condition and the chromatic discrimination ellipse was determined. Discrimination ellipses for the rod incremental and decremental pedestals were measured at eight angles between $0^{\circ}-315^{\circ}$ in the normalized cone space.

\section{Results}

Fig. 2 shows the chromatic discrimination data for the no pedestal (gray symbols), rod incremental (open symbols), and rod decremental (closed symbols) pedestals at $2 \mathrm{Td}$ for both observers, in the relative cone Troland space (left panels) and in the normalized cone space (right panels). In the relative Troland space, the differences among the three conditions appear small, but can be better visualized in the normalized cone space. Clearly, in the absence of rod contrast, the major axis of the discrimination ellipse had a $45^{\circ}$ angle in the normalized cone space. Rod pedestals changed the shape of the discrimination ellipse, but in an asymmetric way. Discrimination along the $0^{\circ}, 270^{\circ}$, and $315^{\circ}$ directions (fourth quadrant of the cone space where L-cone relative to M-cone excitation increased, and S-cone excitation decreased) was degraded with the rod incremental pedestal, but was improved in the presence of the rod decremental pedestal compared with the no pedestal condition. Discrimination along other directions $\left(45^{\circ}-\right.$ $225^{\circ}$ ) did not change appreciably for rod incremental or decremen- 
Interval $1 \quad$ Interval $2 \quad$ Interval 3

Percept
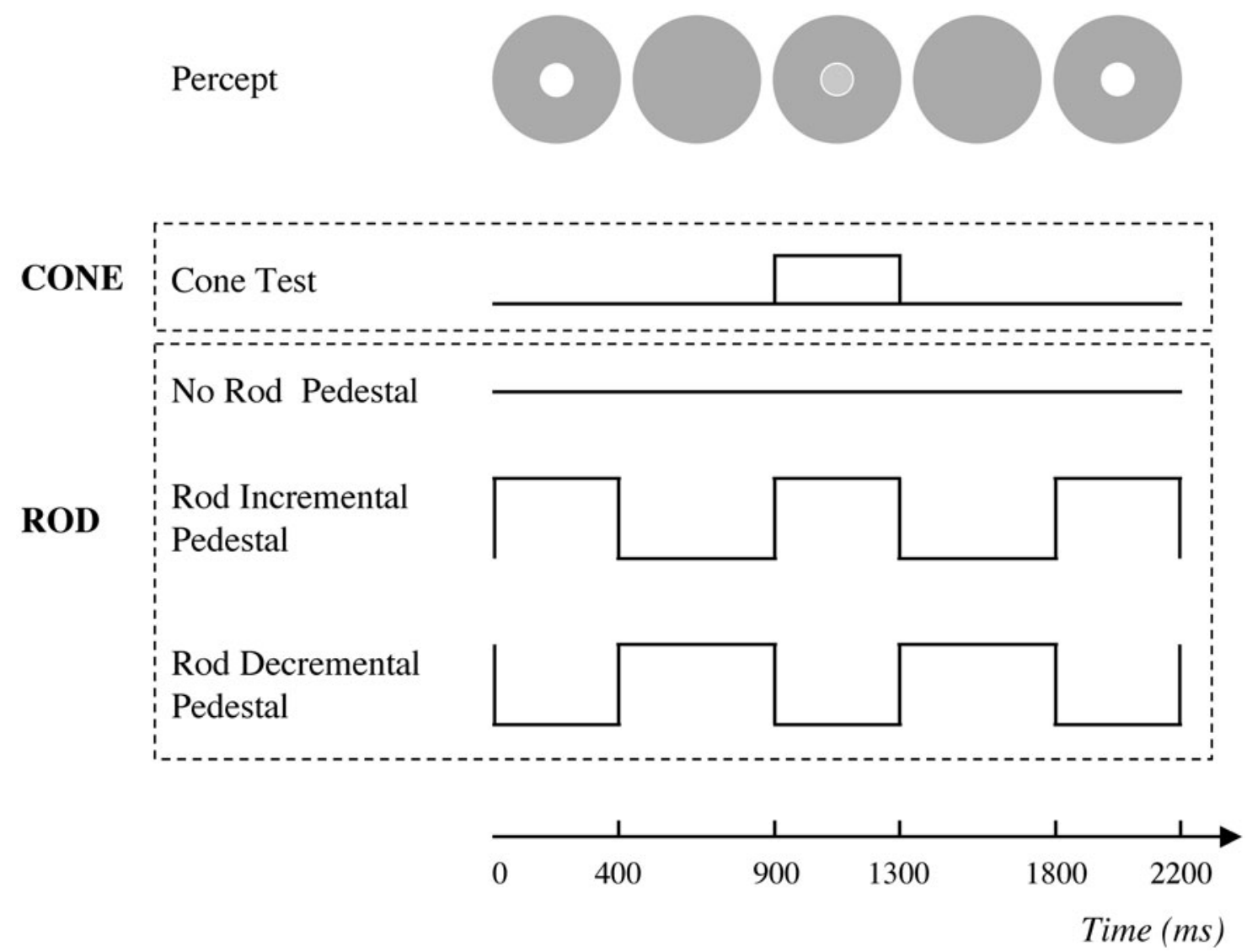

Fig. 1. The schematic representation of the stimulus. A $2^{\circ}$ center within a $13^{\circ}$ surround is set at $7.5^{\circ}$ temporal eccentricity. During a trial, three $400 \mathrm{~ms}$ intervals were presented with $500 \mathrm{~ms}$ separation in between. All three intervals had the same rod pedestal with rod Weber contrast of $0,+20 \%$, or $-20 \%$. One of the intervals (interval 2 in the figure) had a cone chromaticity change along one of the axes in the normalized cone space. The task was to identify the interval and direction of the cone chromaticity change.

tal pedestals, except for the rod decremental pedestal at $225^{\circ}$ for AJZ. Discrimination measurement along the $225^{\circ}$ direction required reducing $\mathrm{L} /(\mathrm{L}+\mathrm{M})$ and $\mathrm{S} /(\mathrm{L}+\mathrm{M})$ simultaneously. For AJZ, however, the rod decrement pedestal altered discrimination along the $270^{\circ}$ direction ( $\mathrm{S}$ decrement) but along the $180^{\circ}$ direction, we infer that the effect of rod decremental pedestal along the $225^{\circ}$ direction was caused by S-decrement discrimination. In summary, rod increments degraded chromatic discrimination and rod decrements improved chromatic discrimination in the fourth quadrant of the normalized cone space. The primary changes in the discrimination ellipse are a shift in the origin and a change in the length of the minor axes.

Experiment 2: Chromatic discrimination in the presence of cone pedestals matched to the appearance of rod pedestals

The second experiment first used a temporal matching technique (Cao et al., 2005) to characterize test color appearance in the presence of a rod pedestal. This was done by equating the color appearance of a rod pedestal to a pedestal in which cone excitations were adjusted. During the stimulus epoch, the rod signal was modulated as a $1 \mathrm{~Hz}$ square wave with steady cone excitations. During the matching epoch, with steady rod excitation, observers adjusted the cone signals $[\mathrm{L} /(\mathrm{L}+\mathrm{M}), \mathrm{S} /(\mathrm{L}+\mathrm{M})$, and $(\mathrm{L}+\mathrm{M})]$ to match the rod percept seen during the stimulus epoch. Observers could toggle freely between the stimulus and matching epochs until a satisfactory match was achieved. Details of the matching paradigm are described in Cao et al. (2005). The matches from three sessions were averaged.

In the second stage of experiment 2, we used the matching results to decompose the rod incremental and decremental pedestals into component $\mathrm{L} /(\mathrm{L}+\mathrm{M}), \mathrm{S} /(\mathrm{L}+\mathrm{M})$, or $(\mathrm{L}+\mathrm{M})$ cone pedestals that matched the appearance of the rod pedestals. We could then measure discrimination with cone pedestals composed of a single component, or a combination of these components. Thus, we measured both $\mathrm{L} / \mathrm{M}$ and $\mathrm{S}$-cone discrimination on cone pedestals composed of the chromatic $\mathrm{L} /(\mathrm{L}+\mathrm{M})$ or $\mathrm{S} /(\mathrm{L}+\mathrm{M})$ component alone, with the luminance $(\mathrm{L}+\mathrm{M})$ component alone, and with combined chromatic and luminance components. Measurements were made along the directions where the rod pedestals altered discrimination threshold (i.e., $0^{\circ}, \mathrm{L} / \mathrm{M}$ increment discrimination, and $270^{\circ}, \mathrm{S}$ decrement discrimination). Discrimination with different cone pedestals was compared to discrimination with 

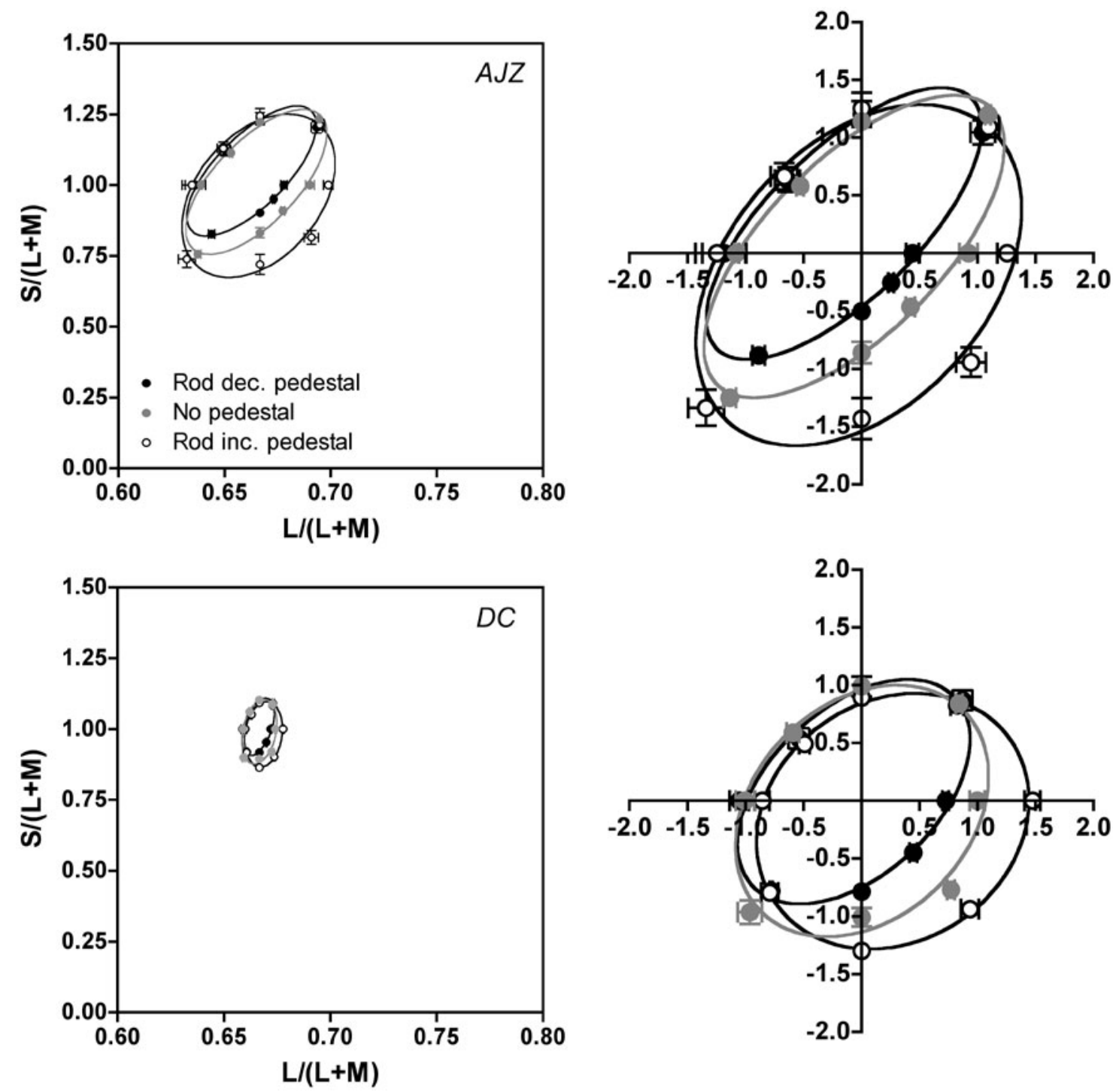

Fig. 2. Chromatic discrimination data with the three pedestal conditions for observer AJZ (top row) and DC (bottom row) in the relative cone Troland cone space (left panels) or a normalized cone space based on threshold along the cardinal axes (right panels).

the rod pedestals to determine if rod contributions to the postreceptoral chromatic and/or luminance pathways were responsible for the altered cone chromatic discrimination.

\section{Results}

With the $20 \%$ rod increment in the test field at $2 \mathrm{Td}$, the average data for observers DC and AJZ (AJZ data in parentheses) show that the cone match required a $0.7 \%(0.6 \%)$ decrease in $\mathrm{L} /(\mathrm{L}+$ $\mathrm{M})$, a $0.8 \%(0.9 \%)$ increase in $\mathrm{S} /(\mathrm{L}+\mathrm{M})$, and an $8.5 \%(8.3 \%)$ increase in $(\mathrm{L}+\mathrm{M})$. With the $20 \%$ rod decrement, the cone match required a $1.1 \%(0.7 \%)$ increase in $\mathrm{L} /(\mathrm{L}+\mathrm{M})$, a $6.2 \%(10.5 \%)$ decrease in $(\mathrm{L}+\mathrm{M})$ and no change $(0.8 \%$ decrease for AJZ) in $\mathrm{S} /(\mathrm{L}+\mathrm{M})$. In other words, at $2 \mathrm{Td}$, with increased rod signals, the test appeared more blue-greenish and brighter; with a decrease in the rod signal, the test appeared more reddish and dimmer.

Fig. 3 shows the results for L/M increment (left panels) and S decrement (right panels) discrimination data (normalized by the discrimination threshold for the no pedestal condition) with appearance matched cone pedestals decomposed into chromatic and luminance components. The leftmost columns in each panel in Fig. 3 show the chromatic discrimination data with the rod pedestals from Experiment 1. Discrimination as a function of rod pedestal contrast increased monotonically, with a lower threshold at the rod decremental contrast for $\mathrm{L} / \mathrm{M}$ increment and $\mathrm{S}$ decrement discrimination. For $\mathrm{L} / \mathrm{M}$ increment discrimination thresholds, the three cone pedestals with a luminance $(\mathrm{L}+\mathrm{M})$ component, including " $(\mathrm{L}+\mathrm{M})$ ", "( $\mathrm{L}+\mathrm{M}) \& \mathrm{~L} /(\mathrm{L}+\mathrm{M})$ ", and "ALL," show a similar pattern, with the no pedestal condition having the highest threshold. With the $\mathrm{L} /(\mathrm{L}+\mathrm{M})$ pedestal however, the $\mathrm{L} / \mathrm{M}$ increment discrimination threshold shows the same monotonic pattern as that with the rod pedestals, indicating rod contributions to the chromatic pathway altered chromatic discrimination. For the $\mathrm{S}$ decrement discrimination $\left(270^{\circ}\right)$, the data with the cone pedestals containing a luminance component, including " $(\mathrm{L}+\mathrm{M})$ ", " $(\mathrm{L}+$ $\mathrm{M})$ and $\mathrm{S} /(\mathrm{L}+\mathrm{M})$ ", and "ALL," were all similar to that with rod 
L/M Increment Discrimination

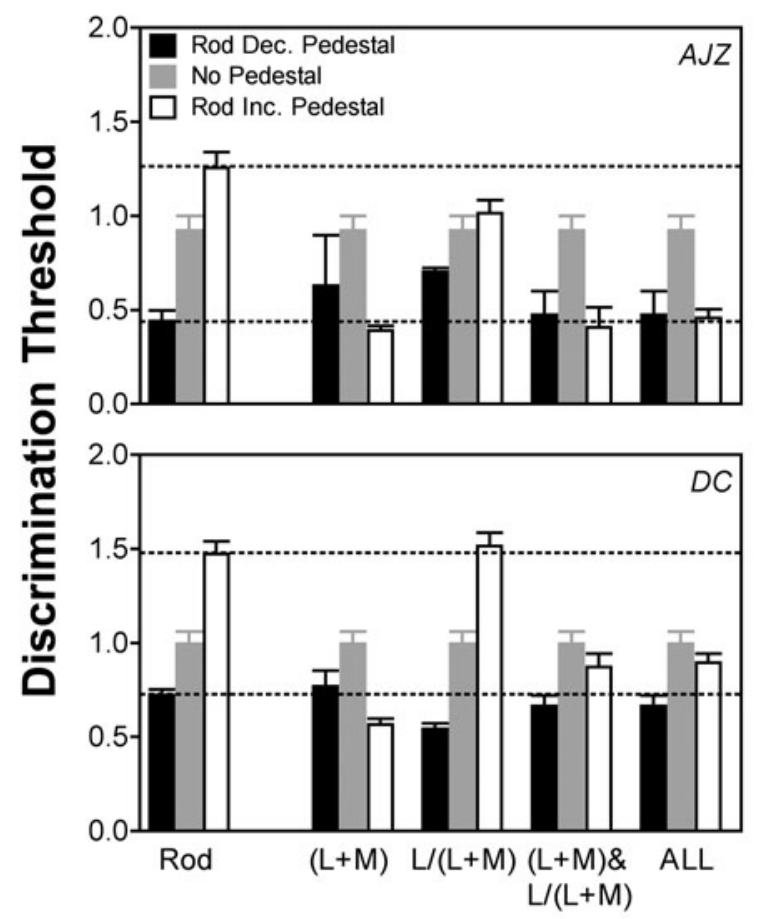

Pedestals
S Decrement Discrimination
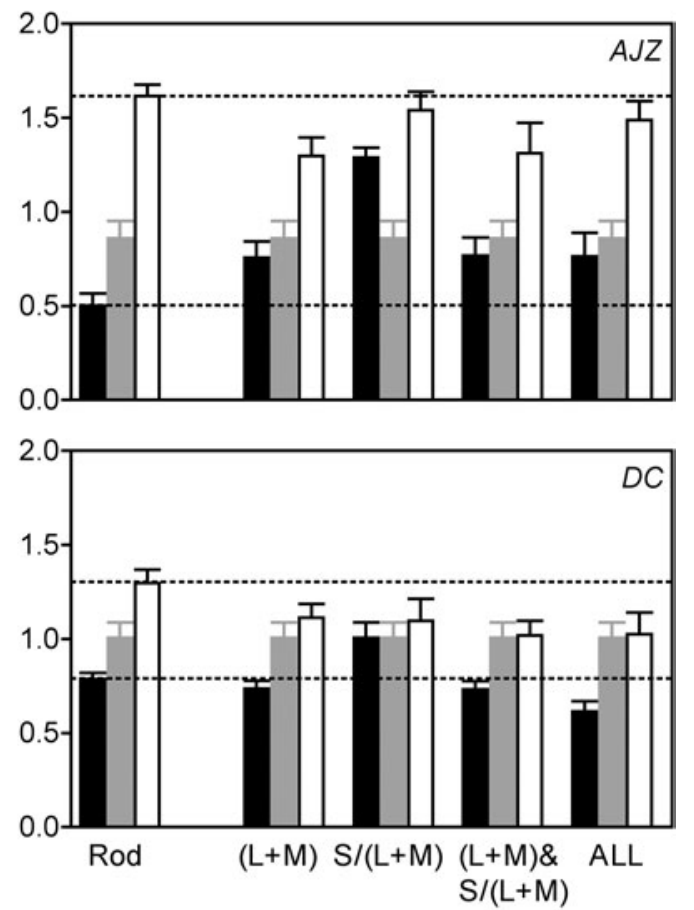

Pedestals

Fig. 3. L/M increment (left panels) and S decrement (right panels) discrimination data with cone pedestals that matched the appearance of the rod pedestals and the chromatic or luminance components of these cone pedestals for observer AJZ (top row) and DC (bottom row). For L/M discrimination, four cone pedestals were used: " $(\mathrm{L}+\mathrm{M})$ " for $(\mathrm{L}+\mathrm{M})$ component alone, " $\mathrm{L} /(\mathrm{L}+\mathrm{M})$ " for $\mathrm{L} /(\mathrm{L}+\mathrm{M})$ component alone, " $(\mathrm{L}+\mathrm{M})$ and $\mathrm{L} /(\mathrm{L}+\mathrm{M})$ " for the combination of $(\mathrm{L}+\mathrm{M})$ and $\mathrm{L} /(\mathrm{L}+\mathrm{M})$ components, "ALL" for the combination of $(\mathrm{L}+\mathrm{M}), \mathrm{L} /(\mathrm{L}+\mathrm{M})$, and $\mathrm{S} /(\mathrm{L}+\mathrm{M})$ components. For $\mathrm{S}$ discrimination, four pedestals were used: " $(\mathrm{L}+\mathrm{M})$ " for $(\mathrm{L}+\mathrm{M})$ component alone, "S/(L+M)" for $\mathrm{S} /(\mathrm{L}+\mathrm{M})$ component alone, " $(\mathrm{L}+\mathrm{M})$ and $\mathrm{S} /(\mathrm{L}+$ $M)$ " for the combination of $(L+M)$ and $S /(L+M)$ components, "ALL" for the combination of $(L+M), L /(L+M)$, and $S /(L+M)$ components. The dashed lines indicate the threshold with the rod decremental or incremental pedestals.

pedestal (right column, Fig. 3), and we infer that rod contribution to the luminance pathway contributed to the observed rod pedestal effect on discrimination.

\section{Discussion}

In the present experiments, rods produced asymmetric changes in cone chromatic discrimination at mesopic light levels. Rod incremental pedestals degraded discrimination, and rod decremental pedestals improved discrimination for test conditions where Lrelative to $\mathrm{M}$-cone excitation increased, and $\mathrm{S}$-cone excitation decreased. The chromatic discrimination threshold pattern with rod pedestals can be characterized as rod contributions to the chromatic (PC-) pathways for $\mathrm{L} / \mathrm{M}$ increment discrimination, or to the luminance (MC-) pathway for $\mathrm{S}$ decrement discrimination.

In an analysis of literature data on chromatic discrimination, Nagy et al. (1987) reported that the majority of the 184 discrimination ellipses analyzed had an orientation of $135^{\circ}$ in the normalized cone space. In contrast, we found that the chromatic discrimination ellipse for the no pedestal condition had a major axis angle of $45^{\circ}$. Yebra et al. (1994) found the orientation of the discrimination ellipse changed from $135^{\circ}$ to $45^{\circ}$ when the stimulus size was increased from $2^{\circ}$ to $8^{\circ}$. In our case, the use of the parafoveal stimulus presentation and the involvement of rods at a mesopic light level likely contributed to the change in the measured orientation of the discrimination ellipses.

Rod effects on chromatic discrimination are sometimes inferred by comparing discrimination obtained following dark adaptation and cone-plateau conditions following the offset a bright adapting light (Stabell \& Stabell, 1977b; Nagy \& Doyal, 1993; Knight et al., 1998). It has been reported that dark-adaptation impairs both L/M discrimination (Nagy \& Doyal, 1993) and S discrimination (Knight et al., 1998). With our instrumentation and experimental conditions, the size of the discrimination ellipse changed when the test stimulus was presented with a rod incremental or decremental pedestal. Discrimination was degraded with the rod incremental pedestal but improved with the decremental pedestal only in the fourth quadrant $\left(0^{\circ}, 270^{\circ}\right.$, and $\left.315^{\circ}\right)$. Knight et al. (2001) reported that rod activity degraded S-decrement discrimination but had virtually no effect on S-increment discrimination, comparable to our findings with the rod incremental pedestal. The fourth quadrant in the normalized cone space reflects higher $L$ relative to $M$ excitation and lower $\mathrm{S}$ excitation. The rod contribution to color perception parallels the discrimination findings here, with rod increments leading to a more blue-greenish percept and rod decrements to a more reddish percept (Cao et al., 2005). It was speculated (Stabell \& Stabell, 1977a) that rod effects on chromatic discrimination might be mediated by a higher level mechanism 
that depends on color appearance, because wavelength discrimination was improved at long wavelengths but degraded at short and middle wavelengths. This proposal does not explain our discrimination data since cone pedestals that matched the appearance of rod pedestals did not yield equivalent results to the rod pedestals (Fig. 3; compare "rod" pedestals to the "ALL" cone pedestal condition that equates the appearance of the rod pedestals).

At photopic light levels where the rod response is attenuated, $\mathrm{L} / \mathrm{M}$ and S-cone discriminations are best at the adapting chromaticity, and degrade with increasing chromatic contrast between the test and adapting field (Zele et al., 2006; Cao et al., 2008). Discrimination can be described by a V-shape in a plot of log threshold as a function of log pedestal L- or S-Tds (for a review see Pokorny \& Smith, 2004). Discrimination along the incremental and decremental directions is quite symmetrical (e.g., Smith et al., 2000; Zele et al., 2006; Cao et al., 2008). Here, with the L/(L + $\mathrm{M})$ cone pedestal we observed a monotonic relationship between discrimination threshold and the chromatic contrast between the test and surround (Fig. 3), indicating a rod effect is present at $2 \mathrm{Td}$. It follows that rod effects on chromatic discrimination can be observed in the presence of rod pedestals and $\mathrm{L} /(\mathrm{L}+\mathrm{M})$ pedestals at mesopic light levels.

The second experiment was designed to evaluate whether changes in cone chromatic discrimination in the presence of incremental or decremental rod pedestals was due to rod contributions to the post-receptoral chromatic and/or luminance pathways. From the rod/cone color appearance matches, we may infer strong rod input to the luminance pathway $(\mathrm{L}+\mathrm{M})$, weak or no input to the chromatic (PC- or KC-) pathway. Cone incremental or decremental pedestals with a luminance component had similar threshold patterns for both $\mathrm{L} / \mathrm{M}$ increment and $\mathrm{S}$ decrement discrimination, suggesting that when chromatic and luminance components in the cone pedestals were presented simultaneously, the luminance signals suppressed chromatic signals. The luminance signals from rod pedestals however, only suppressed chromatic signals in $\mathrm{S}$ decrement discrimination, not in $\mathrm{L} / \mathrm{M}$ increment discrimination. For rod and cone pedestals, the chromatic discrimination results indicated different interaction patterns between post-receptoral luminance and chromatic pathways.

\section{Acknowledgments}

Supported by National Eye Institute grant EY00901-35, Australian Research Council Discovery Project DP0773544 and a Research to Prevent Blindness Challenge Grant. We thank Jules Quinlan for technical assistance with the optical system and Linda Glennie for technical assistance in computer programming.

\section{References}

BucK, S.L. (2004). Rod-cone interaction in human vision. In The Visual Neuroscience, vol. 1, ed. Chalupa, L.M. \& Werner, J.S., pp. 863-878. Cambridge, MA: MIT Press.
CaO, D., Pokorny, J. \& SMith, V.C. (2005). Matching rod percepts with cone stimuli. Vision Research 45, 2119-2128.

CaO, D., Zele, A.J. \& Pokorny, J. (2006). Dark-adapted rod suppression of cone flicker detection: Evaluation of receptoral and postreceptoral interactions. Visual Neuroscience 23, 531-537.

CaO, D., Zele, A.J. \& PoKorny, J. (2007). Linking impulse response functions to reaction time: Rod and cone reaction time data and a computational model. Vision Research 47, 1060-1074.

Cao, D., Zele, A.J., Smith, V.C. \& Pokorny, J. (2008). S-cone discrimination for stimuli with spatial and temporal chromatic contrast. Visual Neuroscience 25, 349-354.

Gilbert, M. (1950). Colour perception in parafoveal vision. Proceedings of the Physical Society (London) B63, 83-89.

Knight, R., Buck, S.L., Fowler, G.A. \& NGuyen, A. (1998). Rods affect S-cone discrimination on the Farnsworth-Munsell 100-hue test. Vision Research 38, 3477-3481.

Knight, R., Buck, S.L. \& Pereverzeva, M. (2001). Stimulus size affects influence on Tritan chromatic discrimination. Color Research and Application 26, S65-S68.

LythGOE, R. (1931). Dark-adaptation and the peripheral colour sensations of normal subjects. British Journal of Ophthalmology 15, 193-210.

MacLeod, D.I.A. \& Boynton, R.M. (1979). Chromaticity diagram showing cone excitation by stimuli of equal luminance. Journal of the Optical Society of America 69, 1183-1185.

NAGY, A.L. \& Doyal, J.A. (1993). Red-green color discrimination as a function of stimulus field size in peripheral vision. Journal of Optical Society of America, A 10, 1147-1156.

Nagy, A.L., Eskew, R.T. \& Boynton, R.M. (1987). Analysis of colormatching ellipses in a cone-excitation space. Journal of the Optical Society of America, A 4, 756-768.

Pokorny, J. \& Smith, V.C. (2004). Chromatic Discrimination. In The Visual Neuroscience, vol. 2, ed. Chalupa, L.M. \& Werner, J.S., pp. 908923. Cambridge, MA: MIT Press.

Pokorny, J., Smithson, H. \& Quinlan, J. (2004). Photostimulator allowing independent control of rods and the three cone types. Visual Neuroscience 21, 263-267.

Puts, M.J.H., Pokorny, J., Quinlan, J. \& Glennie, L. (2005). Audiophile hardware in vision science; the soundcard as a digital to analog converter. Journal of Neuroscience Methods 142, 77-81.

Shapiro, A.G., Pokorny, J. \& SMith, V.C. (1996). Cone-Rod receptor spaces, with illustrations that use CRT phosphor and light-emittingdiode spectra. Journal of the Optical Society of America A 13, 2319-2328.

Smith, V.C. \& Pokorny, J. (1996). The design and use of a cone chromaticity space. Color Research and Application 21, 375-383.

Smith, V.C., PoKorny, J. \& Sun, H. (2000). Chromatic contrast discrimination: Data and prediction for stimuli varying in $\mathrm{L}$ and $\mathrm{M}$ cone excitation. Color Research and Application 25, 105-115.

Stabell, B. \& Stabell, U. (1977a). The chromaticity coordinates from spectrum colours of extrafoveal cones. Vision Research 17, 1091-1094.

Stabell, U. \& Stabell, B. (1977b). Wavelength discrimination of peripheral cones and its change with rod intrusion. Vision Research 17, 423-426.

Sun, H., Pokorny, J. \& Smith, V.C. (2001). Brightness Induction from rods. Journal of Vision 1, 32-41.

Swanson, W.H., Ueno, T., Smith, V.C. \& Pokorny, J. (1987). Temporal modulation sensitivity and pulse detection thresholds for chromatic and luminance perturbations. Journal of the Optical Society of America A 4 , 1992-2005.

Yebra, A., Garcia, J.A. \& Romero, J. (1994). Color discrimination data for 2-degrees and 8-degrees and normalized ellipses. Journal of OpticsNouvelle Revue D Optique 25, 231-242.

Zele, A.J., Smith, V.C. \& Pokorny, J. (2006). Spatial and temporal chromatic contrast: Effect on chromatic contrast discrimination for stimuli varying in L- and M-cone excitation. Visual Neuroscience 23, 495-501. 